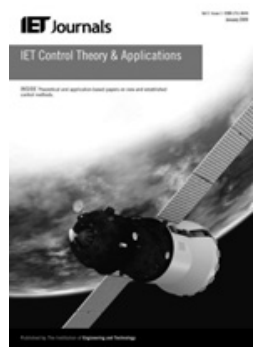

ISSN 1751-8644

\title{
Bounded control of network connectivity in multi-agent systems
}

\author{
D.V. Dimarogonas ${ }^{1}$ K.H. Johansson ${ }^{2}$ \\ ${ }^{1}$ Laboratory for Information and Decision Systems, Massachusetts Institute of Technology, Cambridge, MA, USA \\ ${ }^{2}$ KTH ACCESS Linnaeus Center, School of Electrical Engineering, Royal Institute of Technology (KTH), Stockholm, Sweden \\ E-mail: ddimar@mit.edu
}

Abstract: A distributed control law that guarantees connectivity maintenance in a network of multiple mobile agents is presented. The control law, which lets the agents perform formation manoeuvres, respects sensor limitations by allowing each agent to only take into account agents within its sensing radius. In contrast to previous approaches to the problem, the proposed control law does not attain infinite values whenever an edge of the communication graph tends to be lost. This is achieved via the use of decentralised navigation functions, which are bounded potential fields. The navigation functions are defined to take into account the connectivity maintenance objective. The authors first treat the case of connectivity maintenance for a static communication graph and then extend the result to the case of dynamic graphs. The results are illustrated on a formation control problem.

\section{Introduction}

Recent work on multi-agent cooperative control has paid much attention to consensus and formation control problems. The consensus problem is on the convergence of agents to a common point through a distributed control law supported by a communication network [1-3]. In formation control, agents aim to converge to a specified pattern. The desired formation can be either static [4] or moving [5]. A common assumption in these distributed control problems is the connectedness of the underlying network, that is, that there exists a path connecting any pair of nodes in the network. Although this seems a valid assumption for static wired communication networks, it is not realistic in the case of mobile networks, where communication between nodes usually is distance dependent. Motivated by this fact, a number of recent papers [6-11] consider the distributed connectivity maintenance problem. A common approach in these references is the use of 'unbounded' potential fields that force agents that constitute a distance-based communication link, to remain within a certain distance for all time. In particular, allowing the potential force between such pairs of agents to grow unbounded whenever agents tend to move away from the communication threshold distance provides a guarantee for edge maintenance and thus connectivity. In practical situations, however, the use of unbounded actuation is impossible. The analysis of previous algorithms does not guarantee convergence and connectivity maintenance whenever upper bounds on the actuation are imposed; thus, enforcing the control input to attain arbitrarily large values without guaranteed upper bounds when links tend to be lost is a necessity for these algorithms. Using potential fields with guaranteed bounds is hence desirable.

Motivated by the above observation, we present in this paper the first (to the best of our knowledge) control strategy that handles the distributed connectivity maintenance problem using 'bounded' inputs. In particular, we make use of a bounded potential field that resembles the navigation function potential fields introduced in [12], which was later extended to a decentralised framework in $[13,14]$. The proposed bounded control law (i) maintains the edges that are formed based on the initial positions of the agents and (ii) drives the agents to a common point in the state space. An application of the proposed framework to a formation stabilisation problem is also presented. While the considered control problem involves the single integrator rendezvous and formation stabilisation objectives, the proposed methodology can be applied to 
non-holonomic and double integrator systems as well. For the first case, one can replace the unbounded inter-agent potential used in [7] for connectivity maintenance of multiple nonholonomic agents with the bounded potential defined in this paper. For the case of double integrator agents, one can use [15] and replace the inter-agent potential of that paper with the potential function used here. Decentralised control to preserve connectivity in swarm aggregation with unbounded inputs was recently studied by the first author [10]. In that work a more general problem was considered compared to the current paper. On the other hand, for the particular problem of distributed agreement we are able in the current paper to construct a bounded control law that solves the problem. The existence of such an implementable control law is obviously an important achievement beyond the existing results in the literature. Moreover, the analysis when dealing with bounded inputs is far from straightforward, as seen in the different analysis tools used in the current paper with respect to connectivity maintenance unbounded control laws.

The rest of the paper is organised as follows: in Section 2 the problem is presented. Section 3 begins with the matrix analysis tools used in the paper and presents the control law based on the decentralised navigation function. This function helps us in achieving the bounded input connectivity maintenance objective. The case of dynamic edge addition is treated in Section 4, whereas the proposed framework is applied to a formation control problem in Section 5. Computer simulations are included in Section 6 and Section 7 summarises the results of the paper.

\section{Problem formulation}

We consider $N>1$ integrator point agents in the plane, described by kinematics of the form

$$
\dot{q}_{i}=u_{i}
$$

where $q_{i} \in \mathbb{R}^{2}$ denotes the position and $u_{i} \in \mathbb{R}^{2}$ the velocity (control input) for each agent $i \in \mathcal{N}=\{1, \ldots, N\}$.

Each agent has limited sensing capabilities encoded by a sensing disc with radius $d$. Hence each agent is aware only of the positions of agents within its sensing radius. In order to encode the limited communication between the agents, graph theoretic notions are used, for example, [16]. We assume that each agent is assigned with a subset $N_{i} \subset \mathcal{N}$ of the rest of the team, called agent $i$ 's 'communication set'. Inter-agent communication is encoded in terms of a 'communication graph':

Definition 1: The communication graph $\mathcal{G}=\{V, E\}$ is an undirected graph consisting of a set of vertices $V=\mathcal{N}$ and a set of edges, $E=\left\{(i, j) \in \mathcal{N} \times \mathcal{N} \mid i \in N_{j}\right\}$.

Each agent is supposed to know the relative position of all agents it is connected to through $\mathcal{G}$. Our objective is the construction of bounded control laws that drive the agents to an agreement point while maintaining the connectivity properties induced by the inter-agent relative initial positions. The same problem has been treated for integrator agents $[6,17]$ and for non-holonomic agents [7]; however, the control law in both these papers obtained unbounded values whenever pairs of agents that formed an edge tended to leave the sensing zone of one another. Real mobile agents, however, have limited actuator capabilities and hence boundedness of the control inputs is an issue that should not be neglected. In this paper, we propose a framework based on the navigation function approach of [12], which was further explored in $[13,18,19]$. Note that the control law of [12] is by default bounded, and therefore respects the required actuator limitations.

In summary, the problem treated in the sequel can be stated as follows: 'derive bounded decentralised control laws that respect the limited sensing capabilities of each agent, so that all initial edges are maintained, in the sense that all pairs of agents initially forming an edge remain within distance $d$ from one another, and the agents converge to an agreement point'.

\section{Control design for bounded connectivity preserving}

\subsection{Elements from matrix analysis}

In this subsection we review some tools from matrix analysis and algebraic graph theory that we shall use in the stability analysis of the next sections. The following can be found in standard textbooks on algebraic graph theory [16] and matrix analysis [20].

For a graph $\mathcal{G}$ with $N$ vertices the 'adjacency matrix' $A=A(\mathcal{G})=\left(a_{i j}\right)$ is the $N \times N$ matrix given by $a_{i j}=1$, if $(i, j) \in E$, and $a_{i j}=0$, otherwise. If there is an edge (i, $j) \in E$, then $i, j$ are called adjacent. When there is an orientation defined on each edge $(i, j) \in E$, the graph is called directed otherwise it is called undirected. A path of length $r$ from a vertex $i$ to a vertex $j$ is a sequence of $r+1$ distinct vertices starting with $i$ and ending with $j$ such that consecutive vertices are adjacent and respects the orientation of the edges. If there is a path between any two vertices of the graph $\mathcal{G}$, then $\mathcal{G}$ is called 'strongly connected' in the case of directed graph, and 'connected' in the case of undirected graphs. A directed graph has a 'spanning tree' if there exists at least one vertex to which there exists a path from all other vertices. The graph $\mathcal{G}=(V, E)$ corresponding to a real $N \times N$ matrix $M$ is a graph with $N$ vertices indexed by $1, \ldots, N$ such that there is an edge between vertices $i, j \in V$ if and only if $M_{i j} \neq 0$, that is, $(i, j) \in E \Leftrightarrow M_{i j} \neq 0$.

An $N \times N$ real matrix with non-positive off-diagonal elements and zero row sums is called a Metzler matrix. All eigenvalues of a symmetric Metzler matrix are non-negative 
and zero is a trivial eigenvalue [20]. The multiplicity of the zero eigenvalue of a symmetric Metzler matrix is one if and only if the corresponding undirected graph is connected. The corresponding eigenvector is the vector of ones, $\overrightarrow{\mathbf{1}}$. These results were extended to asymmetric Metzler matrices in [21]. In this paper, we use the following corollary of [21] Theorem 1 :

Corollary 1: Assume that the $N \times N$ time-varying matrix $A(t)$ is Metzler for all $t \geq 0$ and that its elements are piecewise continuous and bounded. Assume also that the time-varying graph corresponding to $A(t)$ is strongly connected for all $t \geq 0$. Then the system $\dot{x}(t)=-A(t) x(t)$ converges to consensus, that is, to an equilibrium with all elements of the vector $x$ equal.

In fact, Corollary 1 holds in the weaker case when $A(t)$ corresponds to a graph containing a spanning tree sufficiently often. We use the strong connectedness assumption since this is sufficient for the main result of this paper.

\subsection{Control design and convergence analysis}

Each agent is equipped with a decentralised navigation function-like potential field of the form $\varphi_{i}: \mathbb{R}^{2 N} \rightarrow[0,1]$

$$
\varphi_{i}=\frac{\gamma_{i}}{\left(\gamma_{i}^{k}+G_{i}\right)^{1 / k}}
$$

where $k>0$ is a positive scalar exponent and the maps $\gamma_{i}, G_{i}$ are discussed in the sequel. It is clear that $\varphi_{i}$ is bounded, taking values in $[0,1]$.

Each agent's communication set $N_{i}$ is defined as the set of agents that are initially located within the sensing zone of agent $i$

$$
N_{i}=\left\{j \in \mathcal{N}, j \neq i:\left\|q_{i}(0)-q_{j}(0)\right\|<d\right\}
$$

This definition of $N_{i}$ justifies the fact that the communication graph, as defined in Definition 1 , is undirected, since (3) implies that $i \in N_{j} \Leftrightarrow j \in N_{i}$ for all $i, j \in \mathcal{N}$. By showing that for all pairs of agents $(i, j)$ s.t. $\left\|q_{i}(0)-q_{j}(0)\right\|<d$ the proposed controller guarantees that $\left\|q_{i}(t)-q_{j}(t)\right\|<d$ for all $t>0$, the edges are guaranteed to remain invariant (i.e. agents $i, j$ remain within distance $d$ from one another) and hence the communication graph itself, remains invariant throughout the closed-loop system evolution. This is shown explicitly in the sequel.

In this section $N_{i}$ is hence a static set. The function $\gamma_{i}$ is defined as

$$
\gamma_{i}(q) \triangleq \sum_{j \in N_{i}} \frac{1}{2}\left\|q_{i}-q_{j}\right\|^{2}
$$

where $q=\left[q_{1}^{\mathrm{T}}, \ldots, q_{N}^{\mathrm{T}}\right]^{\mathrm{T}}$, and so it is minimised whenever the agreement objective with respect to agent $i$ has been fulfilled. The function $G_{i}$ is responsible for the maintenance of the initially formed edges and is defined as

$$
G_{i}(q) \triangleq \prod_{j \in N_{i}} \beta_{i j}(q) \triangleq \prod_{j \in N_{i}} \frac{1}{2}\left(d^{2}-\left\|q_{i}-q_{j}\right\|^{2}\right)
$$

The control law of each agent $i$ is now defined as

$$
u_{i}=-K_{i} \frac{\partial \varphi_{i}}{\partial q_{i}}
$$

where $K_{i}>0$ is a positive gain.

Using the notation $\nabla_{i}(\cdot) \triangleq \partial / \partial q_{i}(\cdot)$ for brevity, we can compute

$$
\begin{aligned}
& \frac{\partial \varphi_{i}}{\partial q_{i}} \\
& =\frac{\left(\gamma_{i}^{k}+G_{i}\right)^{1 / k} \nabla_{i} \gamma_{i}-\left(\gamma_{i} / k\right)\left(\gamma_{i}^{k}+G_{i}\right)^{1 / k-1}\left(k \gamma_{i}^{k-1} \nabla_{i} \gamma_{i}+\nabla_{i} G_{i}\right)}{\left(\gamma_{i}^{k}+G_{i}\right)^{2 / k}}
\end{aligned}
$$

so that

$$
\frac{\partial \varphi_{i}}{\partial q_{i}}=\left(\gamma_{i}^{k}+G_{i}\right)^{-1 / k-1}\left(G_{i} \nabla_{i} \gamma_{i}-\frac{\gamma_{i}}{k} \nabla_{i} G_{i}\right)
$$

Note that since $\gamma_{i}$ and $G_{i}$ are never zero simultaneously, the control law is not infinite whenever two agents tend to the distance $d$ from one another. Thus, unlike previous results on closed-loop connectivity maintenance, the design of this paper allows for boundedness of the control laws each time a link between two agents tends to be broken. This is of course due to the fact that the potential field $\varphi_{i}$ is bounded despite that $G_{i} \rightarrow 0$.

The first result of the paper is that this control law forces agents that are initially within distance $d$ from each other to remain within $d$ for all time. Hence, the communication sets $N_{i}$ are static. The result is stated as follows:

Lemma 1: The set $Q \triangleq\left\{q \in \mathbb{R}^{2 N}: G_{i}(q)>0, i \in \mathcal{N}\right\}$ is invariant for the trajectories of the closed-loop system given by (1) and (4).

Proof: Consider $i \in \mathcal{N}$ and a point $q_{0}$ such that $G_{i}\left(q_{0}\right)=0$. Then

$$
\frac{\partial \varphi_{i}}{\partial q_{i}}\left(q_{0}\right)=\left(\gamma_{i}^{k}\left(q_{0}\right)\right)^{-1 / k-1}\left(-\frac{\gamma_{i}\left(q_{0}\right)}{k} \nabla_{i} G_{i}\left(q_{0}\right)\right)
$$

The partial derivative of $G_{i}$ with respect to $q_{i}$ can be computed by

$$
\nabla_{i} G_{i}=\sum_{j \in N_{i}} \bar{\beta}_{i j} \nabla_{i} \beta_{i j}=-\sum_{j \in N_{i}} \bar{\beta}_{i j}\left(q_{i}-q_{j}\right)
$$


where $\bar{\beta}_{i j} \triangleq \prod_{\substack{i \in N_{i} \\ l \neq j}} \beta_{i l}$. Since $G_{i}\left(q_{0}\right)=0$, then $\beta_{i j}\left(q_{0}\right)=0$ for at least one $j \in N_{i}$. If there exists $j \in N_{i}$ for which $\beta_{i j}\left(q_{0}\right)=0$ and $\beta_{i k}\left(q_{0}\right) \neq 0$, for all $k \in N_{i}, k \neq j$, then $\bar{\beta}_{i j}\left(q_{0}\right)>0$. Hence, in this case $\partial \varphi_{i} / \partial q_{i}$ is non-singular at $q_{0}$. Since $\varphi_{i}$ is also smooth, the result in this case follows from the Implicit Function Theorem. The negated gradient motion $-\partial \varphi_{i} / \partial q_{i}$ is normal to the surface $G_{i}=0$ and hence points towards the set $Q$. When there exists at least two agents $k, j \in N_{i}, k \neq j$ for which $\beta_{i j}\left(q_{0}\right)=\beta_{i k}\left(q_{0}\right)=0$, then $\left(\partial \varphi_{i} / \partial q_{i}\right)\left(q_{0}\right)=0 . \quad$ But $\varphi_{i}: \mathbb{R}^{2 N} \rightarrow[0,1] \quad$ and $\varphi_{i}\left(q_{0}\right)=\gamma_{i}\left(q_{0}\right) /\left(\gamma_{i}^{k}\left(q_{0}\right)+G_{i}\left(q_{0}\right)\right)^{1 / k}=1$, which means that at $q_{0}, \varphi_{i}$ achieves its maximum. However, since the set of initial conditions is open, and no open set of initial conditions can be attracted to the maxima of $\varphi_{i}$ along the negative gradient motion $-\partial \varphi_{i} / \partial q_{i}$ [18], we conclude that $Q$ is invariant.

Note that the function $\gamma_{i}$ can encode any cooperative task apart from the agreement case of this section. This is for example the case of the next section, where formation convergence instead of agreement is encoded by the goal function. Thus Lemma 1 holds irrespective of $\gamma_{i}$.

We hence showed that whenever the system starts within the set $Q$ it remains within this set for all time. Since $G_{i}$ tends to zero whenever an initially formed edge is bound to break, that is, whenever an agent $j \in N_{i}$ tends to leave the sensing radius of $i$, the invariance of $Q$ implies that all agents initially located within distance $d$ from $i$, remain within this distance for all time.

We note that the proposed control law guarantees invariance of $Q$, for a large class of functions $G_{i}$. In the navigation function framework of $[12-14,20]$ the set $G_{i}$ was defined as the collision free set for robot $i$, which guaranteed collision avoidance. Hence the statement and proof of Lemma 1 is similar to the corresponding results of those papers, with the difference being that $G_{i}$ in the current paper establishes edge maintenance.

What is left to show is that the control law (4) leads to agreement in the case of an initially connected communication graph. This is proven by the following theorem:

Theorem 1: Consider the multi-agent control system (1) and (4). Suppose that the initially formed communication graph under ruling (3) is connected and that $G_{i}(q(0))>0$ for all $i \in \mathcal{N}$. Then the agents reach a common point, that is, $q_{i}(t) \rightarrow q^{*}$ for all $i$ and some $q^{*} \in \mathbb{R}^{2}$.

Proof: The partial derivative with respect to $q_{i}$ of the functions $\gamma_{i}$ is

$$
\nabla_{i} \gamma_{i}=\sum_{j \in N_{i}}\left(q_{i}-q_{j}\right)
$$

From the proof of Lemma 1 we have $\nabla_{i} G_{i}=-\sum_{j \in N_{i}} \bar{\beta}_{i j}\left(q_{i}-q_{j}\right)$, where $\bar{\beta}_{i j} \triangleq \prod_{\substack{l \in N_{i} \\ l \neq j}} \beta_{i l}$. We
then have

$$
\begin{aligned}
G_{i} \nabla_{i} \gamma_{i}-\frac{\gamma_{i}}{k} \nabla_{i} G_{i} & =G_{i} \sum_{j \in N_{i}}\left(q_{i}-q_{j}\right)+\frac{\gamma_{i}}{k} \sum_{j \in N_{i}} \bar{\beta}_{i j}\left(q_{i}-q_{j}\right) \\
& =\sum_{j \in N_{i}} \bar{\beta}_{i j}\left(\beta_{i j}+\frac{\gamma_{i}}{k}\right)\left(q_{i}-q_{j}\right) \\
& =\sum_{j \in N_{i}} \pi_{i j}\left(q_{i}-q_{j}\right)
\end{aligned}
$$

where $\pi_{i j} \triangleq \bar{\beta}_{i j}\left(\beta_{i j}+\left(\gamma_{i} / k\right)\right)$. Note that while in general $\pi_{i j} \neq \pi_{j i}$, Lemma 1 guarantees that $\pi_{i j}>0$ and $\pi_{j i}>0$, for all $j \in N_{i}$.

The closed-loop dynamics of each agent are given by

$$
\begin{aligned}
\dot{q}_{i} & =-K_{i}\left(\gamma_{i}^{k}+G_{i}\right)^{-1 / k-1} \sum_{j \in N_{i}} \pi_{i j}\left(q_{i}-q_{j}\right) \\
& =-\sum_{j \in N_{i}} \mu_{i} \pi_{i j}\left(q_{i}-q_{j}\right)
\end{aligned}
$$

with $\mu_{i} \triangleq K_{i}\left(\gamma_{i}^{k}+G_{i}\right)^{-1 / k-1}>0$.

The closed-loop system can now be written in stack vector form as

$$
\dot{q}=-\left(P(q) \otimes I_{2}\right) q
$$

where the matrix $P(q)$ is defined as

$$
P_{i j}(q)= \begin{cases}\sum_{j \in N_{i}} \mu_{i}(q) \pi_{i j}(q), & i=j \\ -\mu_{i}(q) \pi_{i j}(q), & j \in N_{i} \\ 0, & \text { otherwise }\end{cases}
$$

and $I_{2}$ is the two-dimensional unit matrix. The matrix $P$ is a Metzler matrix with zero row sums. The stability properties of (5) have been studied in [21]: since $P_{i j}<0$ implies $P_{j i}<0$ for all $j \in N_{i}$, the directed graph corresponding to $P$ is strongly connected if and only if the initially formed undirected communication graph is connected. In essence, if the agents start from the set $Q$, the matrix $P$ trivially satisfies the conditions of Corollary 1 (and thus of [21] Theorem 1) provided that the initially formed communication graph is connected. Hence if the graph is initially connected, the agents reach a common value in the state space.

Theorem 1 guarantees that all initially formed edges are maintained. In essence, the communication graph that is formed based on the initial relative positions of the agents is invariant and the proposed bounded control law drives the multi-agent team to agreement.

The control law can be further elaborated to take into account possible disturbances and parameter uncertainties. This is due to the fact that it is a feedback control law. 
For example, when there is uncertainty in the computation of the sensing radius $d$, we can use a smaller value $d^{\prime}<d$ in the control design of Section 3.2. In some cases, it is desirable to take into account possible additions of edges, each time a new agent enters the sensing zone of another. This is the topic of the next section.

\section{Dynamic edge addition}

The previous section involved the case where the communication graph considered was static, that is, no new edges were added whenever an agent, not initially located within the sensing zone of another agent, entered its sensing zone. In practical situations, however, it is more interesting to consider the creation of new edges whenever an agent enters the sensing zone of another. This naturally leads to a faster convergence rate. Allowing each agent to consider every agent as a neighbour once it enters the neighbour set leads to an increased communication cost, in the sense that each agent will have to keep track of more functions of the form $\beta_{i j}$. Hence there is a trade-off between faster convergence rate and communication cost. In this section, we consider the dynamic graph formulation for the same agent model as previously. We encode the trade-off discussed previously by allowing each agent to have a maximum number of neighbours $M \leq N$. Each agent is allowed to include up to a certain number of agents, $M$, in its control law. The number $M$ can be decided off-line according to an optimisation procedure that takes into account this trade-off. The main result of this section shows that in fact each agent will have exactly $M$ neighbours at steady state for the proposed distributed control law.

In this section, the neighbouring set of each agent $i$ is initially defined as the set of agents that belong to its sensing zone at the initial time $t=0$

$$
N_{i}(0)=\left\{j \in \mathcal{N}, j \neq i:\left\|q_{i}(0)-q_{j}(0)\right\|<d\right\}
$$

We assume that a new communication link for agent $i$ is created each time a new agent enters a subset of the sensing zone of $i$. In particular, we define the set

$$
\begin{aligned}
N_{i}^{*}(t)= & \left\{j \in \mathcal{N}, j \neq i:\left(j \notin N_{i}\left(t^{-}\right)\right)\right. \\
& \left.\wedge\left(\left\|q_{i}(t)-q_{j}(t)\right\|<d-\varepsilon\right)\right\}
\end{aligned}
$$

where $N_{i}\left(t^{-}\right)$denotes the left-hand limit of $N(t)$ at time $t$ and $\varepsilon$ is a small positive scalar. It is obvious that $N_{i}^{*}(t) \subseteq N_{i}(t)$.

Assumption 1: The parameter $M$ is chosen so that there exists a realisation of the graph with $N$ vertices where all the vertices have exactly $M$ adjacent vertices.

This assumption is a viability condition on the edge addition ruling that will now be defined. In particular, the communication set of each agent $i$ is updated according to the following rule

$$
N_{i}(t)= \begin{cases}N_{i}\left(t^{-}\right) \cup N_{i}^{*}(t) & \text { if }\left|N_{i}\left(t^{-}\right)\right|<M \\ N_{i}\left(t^{-}\right) & \text {otherwise }\end{cases}
$$

The function $G_{i}$ is defined as earlier

$$
G_{i}=\prod_{j \in N_{i}(t)} \frac{1}{2}\left(d^{2}-\left\|q_{i}-q_{j}\right\|^{2}\right)
$$

as is the control law of each agent

$$
u_{i}=-\sum_{j \in N_{i}(t)} \mu_{i} \pi_{i j}\left(q_{i}-q_{j}\right)
$$

which is updated whenever a new agent enters the neighbouring set $N_{i}$ of agent $i$.

It is easily seen that whenever a new edge is created the function $G_{i}$ remains strictly positive, so the edge maintenance result of Lemma 1 still holds and hence whenever two agents form a new link, that is, are at a distance less than $d-\varepsilon$ for the first time, they will remain within distance $d$. Thus, the definition of dynamic edge addition respects the limited sensing capabilities of all agents. Thus, once a new edge is created it is never lost.

In the degenerate case where two or more agents enter the set $N_{i}^{*}(t)$ simultaneously, that is, at exactly the same time instant $t$, the set $N_{i}(t)$ can result to have more than $M$ agents. In order to exclude this degenerate case, we make the following assumption:

Assumption 2: For all $i \in \mathcal{N}$, the set of initial conditions for which there exists $j, k \notin N_{i}(0), j, k \neq i$, and $t>0$, such that

$$
\begin{gathered}
\left(j, k \notin N_{i}(0)\right) \wedge\left(\left\|q_{i}(t)-q_{j}(t)\right\|<d-\varepsilon\right) \\
\wedge\left(\left\|q_{i}(t)-q_{k}(t)\right\|<d-\varepsilon\right)
\end{gathered}
$$

has measure zero.

Assumption 2 can be relaxed by incorporating the agents with a selection mechanism that chooses which links to add when two or more agents enter the set $N_{i}^{*}(t)$ simultaneously. To study the consequences of such a selection mechanism is beyond the scope of this paper.

We are now ready to state the main result of this section:

Theorem 2: Consider the multi-agent control system (1) and (8). Suppose that the initially formed communication graph under ruling (6) is connected and that $G_{i}(q(0))>0$ for all $i \in \mathcal{N}$. Assume moreover that $N_{i}(0) \leq M$ for all $i \in \mathcal{N}$ and that Assumptions 1 and 2 hold. Then, for almost all initial conditions, the agents reach a common point, that is, $q_{i}(t) \rightarrow q^{*}$ for all $i$ and some $q^{*} \in \mathbb{R}^{2}$. Moreover, $\lim _{t \rightarrow \infty}\left|N_{i}(t)\right|=M, \forall i \in \mathcal{N}$, that is, all agents have exactly $M$ neighbours at steady state. 
Proof: The proof follows the same arguments as the corresponding one in [6]. If no new edges are added, then Theorem 1 holds, that is, all agents converge to the same point in the workspace. However, this means that all agents eventually come to a distance $d-\varepsilon$ from one another. It is then evident that all agents attain the maximum number of neighbours, $M$, allowed by the edge addition ruling (7), while converging to a common point in the state space.

This theorem guarantees that connectedness is maintained while each agent may add new neighbours until the number of neighbours of each agent is exactly $M$. The result can be slightly modified letting the agents have different parameters $M$. Note that the control law (8) satisfies the actuator boundedness requirement.

Some further remarks regarding the parameter $M$ are in order. Note first that the case when $N_{i}(0)>M$ for some $i$ is excluded from the statement of Theorem 2. In order to incorporate this case, we can chose $M$ to be equal to the maximum number of initial neighbours an agent has, that is, $M=\max _{i \in \mathcal{N}}\left|N_{i}(0)\right|$. Note also that the initial graph should always be connected, as stated in Theorem 2, for the theorem to hold. The case of having a fixed $M$ and choosing the initial neighbours in order to render the initial graph connected is beyond the scope of the current paper whose focus is on the design and stability analysis of the bounded connectivity maintenance control laws. How to set $M$ and choose initial neighbours in general can be seen as an open problem of multi-agent control. Moreover, as already mentioned, the choice of $M$ affects the communication cost and convergence speed trade-off. The case $M=N$ is the extreme that obviously maximises the communication cost. On the other hand, the initial graph being a spanning tree is the other extreme case, which can take place if we modify the result in order to allow the agents to have different parameters $M$, as discussed above. Such trees may be constructed off-line in a distributed way according to [22]. We can then use the static graph formulation of the previous section in order to maintain the tree structure during convergence to agreement. In general, the choice of $M$ can be formulated as an optimisation problem that takes into account the trade-off discussed above and the initial positions. The above issues are beyond the scope of the current paper, whose focus is on the design of bounded control laws for connectivity maintenance.

\section{$5 \quad$ Connectivity preserving formation control}

In this section we revisit the static graph formulation of Section 3 and show how this can be applied to a formation stabilisation problem. The formation stabilisation is such that each agent $i$ converges to a relative position $c_{i j}$ with respect to each of its neighbours $j \in N_{i}$. Thus, a vector $c_{i j} \in \mathbb{R}^{2}$ is associated to each edge $(i, j) \in E$ of the communication graph $\mathcal{G}=(V, E)$, in order to specify the desired inter-agent relative positions in the final formation. The desired formation is called 'feasible' if it belongs to the set $\Phi \triangleq\left\{q \in W \mid q_{i}-q_{j}=c_{i j}, \forall(i, j) \in E\right\}$ of all possible desired formations and this set is non-empty. Only pairs of agents $i, j$ that are initially within the sensing distance $d$ are assigned a desired relative formation vector $c_{i j}$. In order not to violate this communication ruling, the requirement

$$
\left\|c_{i j}\right\|<d, \forall(i, j) \in E
$$

is imposed on all edges of the communication graph.

The formation control law for each agent $i$ is given by

$$
u_{i}=-K_{i} \frac{\partial \varphi_{i}^{\mathrm{f}}}{\partial q_{i}}
$$

where the connectivity preserving navigation function is

$$
\varphi_{i}^{\mathrm{f}}(q) \triangleq \frac{\gamma_{i}^{\mathrm{f}}(q)}{\left(\left(\gamma_{i}^{\mathrm{f}}(q)\right)^{k}+G_{i}^{\mathrm{f}}(q)\right)^{1 / k}}
$$

where

$$
\gamma_{i}^{\mathrm{f}}(q) \triangleq \sum_{j \in N_{i}}\left(\left\|q_{i}-q_{j}-c_{i j}\right\|\right)^{2}
$$

and

$$
G_{i}^{\mathrm{f}}(q) \triangleq \prod_{j \in N_{i}} \beta_{i j}^{\mathrm{f}}(q) \triangleq \prod_{j \in N_{i}} \frac{1}{2}\left(\left(d-\left\|c_{i j}\right\|\right)^{2}-\left\|q_{i}-q_{j}-c_{i j}\right\|^{2}\right)
$$

Similarly to the analysis of the static graph case, the formation control law can be calculated as

$$
u_{i}=-\sum_{j \in N_{i}} \mu_{i}^{\mathrm{f}} \pi_{i j}^{\mathrm{f}}\left(q_{i}-q_{j}-c_{i j}\right)
$$

where

$$
\mu_{i}^{\mathrm{f}} \triangleq K_{i}\left(\left(\gamma_{i}^{\mathrm{f}}\right)^{k}+G_{i}^{\mathrm{f}}\right)^{-1 / k-1}
$$

and

$$
\pi_{i j}^{\mathrm{f}} \triangleq \bar{\beta}_{i j}^{\mathrm{f}}\left(\beta_{i j}^{\mathrm{f}}+\frac{\gamma_{i}^{\mathrm{f}}}{k}\right)
$$

with $\bar{\beta}_{i j}^{\mathrm{f}} \triangleq \prod_{\substack{l \in N_{i} \\ l \neq j}} \beta_{i l}^{\mathrm{f}}$. Lemma 1 has the following counterpart in the formation control case:

Lemma 2: The set $Q^{\mathrm{f}} \triangleq\left\{q \in \mathbb{R}^{2 N}: G_{i}^{\mathrm{f}}(q)>0, i \in \mathcal{N}\right\}$ is invariant for the trajectories of the closed-loop system given by (1) and (10).

Proof: See proof of Lemma 1. 


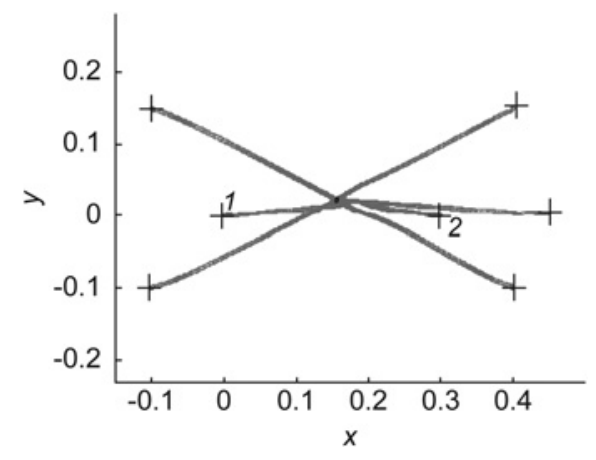

Figure 1 Connectivity preserving state agreement with bounded inputs control law (4) and initial condition rule (3)

We hence conclude that the set $Q^{\mathrm{f}}$ is positively invariant for all agents $i \in \mathcal{N}$. Thus, for each pair of agents that form an edge we have $\left(d-\left\|c_{i j}\right\|\right)^{2}-\left\|q_{i}-q_{j}-c_{i j}\right\|^{2}>0$. By virtue of (9) we have $\left\|c_{i j}\right\|<d$, equation $\left(d-\left\|c_{i j}\right\|\right)^{2}-\left\|q_{i}-q_{j}-c_{i j}\right\|^{2}>0$ does not violate the connectivity preserving ruling $\left\|q_{i}-q_{j}\right\|<d$, since

$$
\begin{aligned}
& \left(d-\left\|c_{i j}\right\|\right)^{2}-\left\|q_{i}-q_{j}-c_{i j}\right\|^{2}>0 \\
& \stackrel{\left\|c_{i j}\right\|<d}{\Rightarrow}\left\|q_{i}-q_{j}-c_{i j}\right\|<d-\left\|c_{i j}\right\| \\
& \Rightarrow\left\|\left|q_{i}-q_{j}\|-\| c_{i j}\|\mid<d-\| c_{i j}\|\Rightarrow\| q_{i}-q_{j} \|<d\right.\right.
\end{aligned}
$$

for all $(i, j) \in E$.
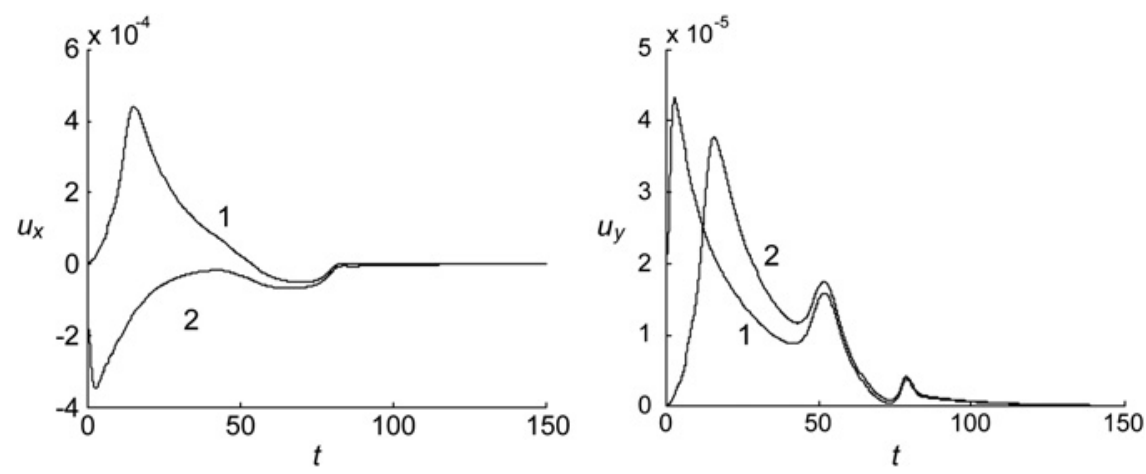

The closed-loop system (13) behaves in the $\tilde{q}$ space as the system (5) behaves in the $q$ space, in the sense that Theorem 1 holds in the $\tilde{q}$ space for the system (13). Thus, (13) reaches a configuration where the elements of the stack vector $\tilde{q}$ are equal, that is, $\lim _{t \rightarrow \infty} \tilde{q}_{i}(t)=$ $\lim _{t \rightarrow \infty} \tilde{q}_{j}(t)=\tilde{q}^{*}$ for all $i, j \in \mathcal{N}$. Thus, $q_{i}-q_{j}=\tilde{q}_{i}-\tilde{q}_{j}+$ $c_{i}-c_{j}=\tilde{q}^{*}-\tilde{q}^{*}+c_{i j}$ so that $q_{i}-q_{j}=c_{i j}, \forall(i, j) \in E$. We conclude that the agents converge to the desired relative configuration. The above observations are summarised in the following theorem:

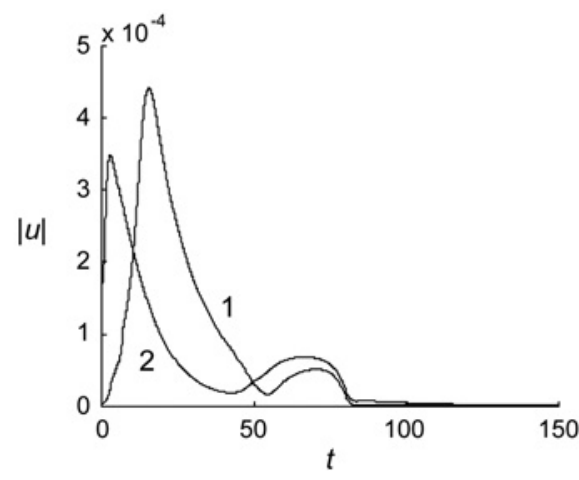

Figure 2 Velocity diagrams

The first two graphs show the plots of the coefficients of the velocities (control inputs) of agents 1 and 2 in the $x, y$ directions whereas the right plot shows the evolution of the velocity norms of the agents 1 and 2 in time 


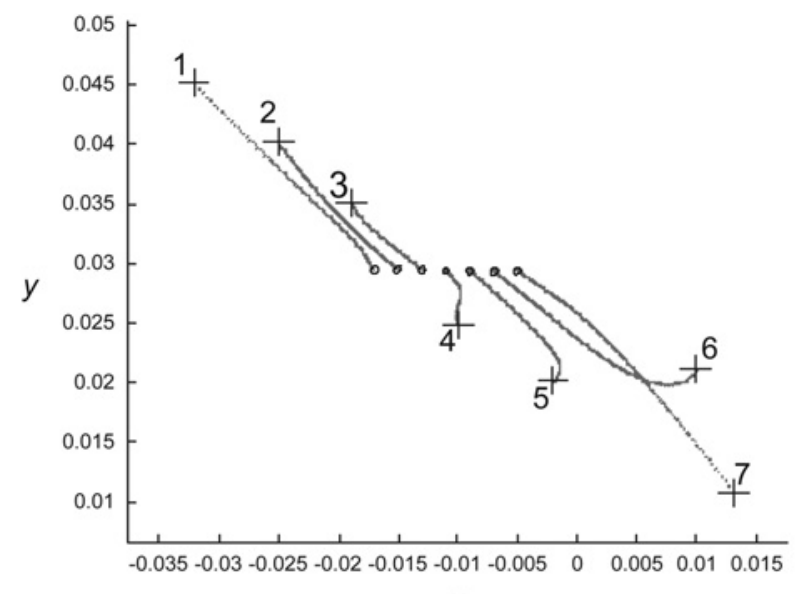

$X$

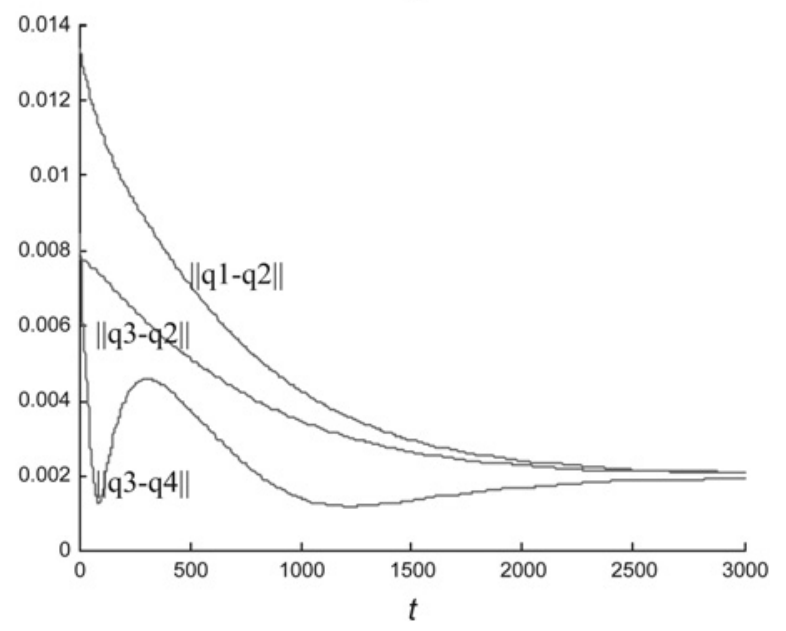

Figure 3 In the top plot, seven agents converge to a line formation under the control (12)

The connectivity of the communication graph formed under the initial condition ruling (3) is preserved. The bottom plot shows the distances of three pairs of agents that form an edge in the initially formed communication graph. All distances converge to the expected value

Theorem 3: Consider the multi-agent system (1) and (10). Suppose that the initially formed communication graph under ruling (3) is connected. Further assume that (9) holds and that the desired formation is feasible. Then the initially formed edges are maintained, that is, pairs of agents initially within a distance $d$ from one another remain within this distance for all time, and the multi-agent team reaches the desired formation.

\section{Examples}

To support the results of the paper, computer simulations are provided in this section.

\subsection{Example 1}

In the first simulation, six agents evolve under the control (4) and the rule (3). The initially formed graph is connected and remains invariant by virtue of Lemma 1. In Fig. 1, the agents' initial positions are marked with a cross. In this example, $d=0.36$. Note that although agents 1 and 2 are initially very close to losing their link due to the existence of the two subgroups on their left- and right-side, respectively, the controller forces them to maintain it. The network stays connected and agents converge to an agreement point in accordance with Theorem 1. The boundedness of the inputs of agents 1 and 2 that tend to break their link is depicted in the velocity diagrams of Fig. 2. The first two graphs show the plots of the coefficients of the velocities (control inputs) of agents 1 and 2 in the $x, y$ directions whereas the right plot shows the evolution of their velocity norms.

\subsection{Example 2}

The next simulation of Fig. 3 presents a formation control example. The seven agents navigate under the control law (12). The communication graph, which is formed based on the initial condition ruling (3), is in fact a line graph. The sensing radius is given by $d=0.025$ while formation objective is convergence to a straight line, where each agent will be at a distance equal to $\left\|c_{i j}\right\|=0.002<d$ from each of its neighbours. In the left plot of Fig. 3, the crosses represent the initial positions of the agents and their final locations are noted by a black circle. Moreover, the trajectory of each agent is the line that connects its initial and final configuration. As witnessed in the plot, the agents eventually reach the desired line formation. The right plot Fig. 3 verifies that the interagent distances do in fact converge to the desired distance $\left\|c_{i j}\right\|=0.002<d$. The figure shows the plot of the inter-agent distances of three pairs of agents that form an edge in the initially formed communication graph. All distances converge to 0.002 , as shown in the figure.

\section{Conclusions}

A distributed control law that guarantees connectivity maintenance in a network of multiple mobile agents was presented. The control law respects the limited sensing capabilities of the agents by allowing each agent to take into account only agents within its sensing radius in the controller implementation. In contrast to previous approaches on the problem, the proposed control law does not attain infinite values whenever an edge tends to be lost, and is therefore a bounded distributed control law. This is achieved via the use of decentralised navigation functions, which are potential fields with guaranteed boundedness and are redefined in this paper in order to take into account the connectivity maintenance objective. We first treated the case of connectivity maintenance in a static initial positionbased communication graph and extended the results to the case of dynamic edge addition. The results were then applied to a formation control problem.

Future work includes extending the results to other primary control objectives as well as taking into account probabilistic models in the inter-agent communication. 


\section{Acknowledgment}

This work was done within TAIS-AURES program (297316-LB704859), funded by the Swedish Governmental Agency for Innovation Systems (VINNOVA) and the Swedish Defence Material Administration (FMV). It was also supported by the Swedish Research Council, the Swedish Foundation for Strategic Research and the EU FeedNetBack STREP FP7 project. A preliminary version of this work appeared in [23].

\section{References}

[1] XIAO L., BOYD S.: 'Fast linear iterations for distributed averaging'. 42nd IEEE Conf. Decision and Control, 2003, pp. 4997-5002

[2] Olfati-SABer R., murray R.M.: 'Consensus problems in networks of agents with switching topology and time-delays', IEEE Trans. Autom. Control, 2004, 49, (9), pp. 1520-1533

[3] FANG L., ANTSAKLIS P.J., TZIMAS A.: 'Asynchronous consensus protocols: preliminary results, simulations and open questions'. 44th IEEE Conf. Decision and Control, 2005, pp. 2194-2199

[4] HSIEH M.A., KUMAR V.: 'Pattern generation with multiple robots'. IEEE Int. Conf. Robotics and Automation, 2006, pp. $2442-2447$

[5] OlfATI-SABER R.: 'Flocking for multi-agent dynamic systems: algorithms and theory', IEEE Trans. Autom. Control, 2006, 51, (3), pp. 401-420

[6] Jl M., Egerstedt M.: 'Connectedness preserving distibuted coordination control over dynamic graphs'. 2005 American Control Conf., 2005, pp. 93-98

[7] DIMAROGONAS D.V., KYRIAKOPOULOS K.J.: 'On the rendezvous problem for multiple nonholonomic agents', IEEE Trans. Autom. Control, 2007, 52, (5), pp. 916-922

[8] ZAVLANOS M.M., PAPPAS G.J.: 'Distributed connectivity control of mobile networks'. 46th IEEE Conf. Decision and Control, 2007, pp. 3591-3596

[9] ZaVlanos M.M., tAHBAZ-SAleHI A., JADbabaie A., PAPPAS G.J.: 'Distributed topology control of dynamic networks'. 2008 American Control Conf., 2008, pp. 2660-2665

[10] DIMARogonas D.V., KYRIAKopoulos K.J.: 'Connectedness preserving distributed swarm aggregation for multiple kinematic robots', IEEE Trans. Robot., 2008, 24, (5), pp. $1213-1223$
[11] degennaro M.C., Jadbabaie A.: 'Decentralized control of connectivity for multi-agent systems'. 45th IEEE Conf. Decision and Control, 2006, pp. 3628-3633

[12] KODITSCHEK D.E., RIMON E.: 'Robot navigation functions on manifolds with boundary', Adv. Appl. Math., 1990, 11, pp. $412-442$

[13] DIMAROGONAS D.V., LOIZOU S.G., KYRIAKOPOULOS K.J., ZAVLANOS M.M.: 'A feedback stabilization and collision avoidance scheme for multiple independent non-point agents', Automatica, 2006, 42, (2), pp. 229-243

[14] TANNER H.G., KUMAR A.: 'Formation stabilization of multiple agents using decentralized navigation functions', in THRUN S., SUKHATME G., SCHAAL S., BROCK O. (EDS.): Robotics: Science and Systems I (MIT Press, 2005, pp. 49-56)

[15] REN W., ATKINS E.: 'Distributed multi-vehicle coordinated control via local information exchange', Int. J. Robust Nonlinear Control, 2007, 17, (10-11), pp. 1002-1033

[16] GODSIL C., ROYLE G.: 'Algebraic graph theory', Graduate Texts in Mathematics (Springer, vol. 207, 2001)

[17] Jl M., EGERSTEDT M.: 'Distributed coordination control of multiagent systems while preserving connectedness', IEEE Trans. Robot., 2007, 23, (4), pp. 693-703

[18] Karagoz C.S., Bozma H.I., Koditschek D.E.: 'Coordinated navigation of multiple independent disk-shaped robots'. Tech. Report no. mscis-07-16, Department of Computer and Information Science, University of Pennsylvania, 2007

[19] LOIZOU S.G., KYRIAKOPOULOS K.J.: 'Closed loop navigation for multiple holonomic vehicles'. Proc. IEEE/RSJ Int. Conf. on Intelligent Robots and Systems, 2002, pp. 2861-2866

[20] lUenberger D.G.: 'Introduction to dynamic systems: theory, models and applications' (John Wiley \& Sons, 1979)

[21] MOREAU L.: 'Stability of continuous-time distributed consensus algorithms'. 43rd IEEE Conf. Decision and Control, 2004, pp. 3998-4003

[22] Gallager R.G., hUMblet P.A., SPIRA M.: 'A distributed algorithm for minimum-weight spanning trees', $A C M$ Trans. Program. Lang. Syst., 1983, 5, (1), pp. 66-77

[23] DIMAROgonAS D.V., JOHANSSON K.H.: 'Decentralized connectivity maintenance in mobile networks with bounded inputs'. IEEE Int. Conf. Robotics and Automation, 2008, pp. 1507-1512 